\title{
TRES PERSPECTIVAS EN TORNO AL USO COMESTIBLE \\ DE LAS INFLORESCENCIAS DE LAS PALMAS PACAY(A) Y CHAPAY(A) \\ EN CHIAPAS, MÉXICO: ENFOQUES PALEOETNOBOTÁNICO, NUTRICIONAL Y LINGÜÍSTICO
}

\author{
LuCERo Meléndez GuadarRama \\ Instituto de Investigaciones Antropológicas \\ Universidad Nacional Autónoma de México \\ Felipe Trabanino \\ Posgrado en Antropología, FFY \\ Universidad Nacional Autónoma de México \\ Adriana Caballero Roque \\ Facultad de Ciencias de la Nutrición y Alimentos \\ Universidad de Ciencias y Artes de Chiapas
}

\begin{abstract}
Resumen:: A partir de evidencia etnográfica actual así como de tres tipos de análisis (paleoetnobotánico, nutricional y linguístico) se muestra la importancia cultural de las inflorescencias de las palmas pacay(a) y chapay(a) con fines comestibles en Chiapas, México. Los resultados del análisis paleoetnobotánico indican la presencia de fitolitos tipo PALMAE recuperados del sedimento del depósito del palacio de Chinikihá, en las cercanías de Palenque. El análisis del contenido nutrimental de las flores de las palmas pacay(a) y chapay(a) muestra cuáles son los nutrientes (proteínas, minerales y vitaminas) que contienen dichas plantas, mismas que complementan la dieta de algunos grupos mayas en la actualidad y, posiblemente, desde el Clásico. Por último, con el análisis linguíístico proponemos que los términos č'ib' (del protomaya "k'iib') y ?ak te? o čitam te? son los términos vernáculos mayas para pacay(a) y chapay(a) respectivamente, y que estos últimos tienen un origen quechua.
\end{abstract}

Palabras clave: Inflorescencias de palmas, paleoetnobotánica, alimentación maya, nutrimentos, lingüística maya.

ABSTRACT: Based on current ethnographic information in conjunction with paleoethnobotanical, nutritional, and linguistic data, this study examines the cultural importance of the inflorescences of palms pacay(a) and chapay(a) with edible purposes in Chiapas, Mexico. Paleoethnobotanical analysis of sediment from Chinikihá's palace, near Palenque, Chiapas, documented the presence of PALMAE phytoliths. Nutritional studies reveal that the flowers of pacay(a) and chapay(a) contain high amounts of proteins, minerals, and vitamins, wich complement the diet of some Maya groups today and, possibly, since Classical times. Lastly, linguistic evidence shows that the terms 'ćib' (from protomayan "k'iib') and Pak te? or čitam te? are the Mayan vernacular names for pacay(a) and chapay(a) and that the latter two have a quechua origen.

KEYwords: Palm inflorescens, paleoethnobotany, nutrition, mayan food, mayan linguistics.

RECEPCIÓN: 13 de marzo de 2012.

ACEPTACIÓN: 18 de junio de 2012. 



\title{
TRES PERSPECTIVAS EN TORNO AL USO COMESTIBLE DE LAS INFLORESCENCIAS DE LAS PALMAS PACAY(A) Y CHAPAY(A) EN CHIAPAS, MÉXICO: ENFOQUES PALEOETNOBOTÁNICO, NUTRICIONAL Y LINGÜÍSTICO ${ }^{1}$
}

\author{
LuCERo Meléndez Guadarrama \\ Instituto de Investigaciones Antropológicas \\ Universidad Nacional Autónoma de México \\ FELIPE TRABANino \\ Posgrado en Antropología, FFYL \\ Universidad Nacional Autónoma de México \\ Adriana Caballero Roque \\ Facultad de Ciencias de la Nutrición y Alimentos \\ Universidad de Ciencias y Artes de Chiapas
}

\section{Introducción}

El siguiente trabajo es una colaboración pluridisciplinaria que contempla tres enfoques en torno al uso de las palmas chapay(a) (Astrocaryum mexicanum) y pacay(a) (Chamaedorea tepejilote) en Chiapas, México: el enfoque paleoetnobotánico, que consiste en la identificación de restos vegetales en contextos arqueológicos con el fin de entender las interacciones ente las sociedades pasadas y su entorno vegetal. Este enfoque se apoya en la etnobotánica para realizar analogías en cuanto al uso de especies vegetales, y al manejo tradicional de recursos vegetales (Pearsall, 2000). Por otra parte, a través del enfoque nutricional se analizan los valores nutritivos de las inflorescencias de las palmas pacay(a) y chapay(a). Y finalmente, el enfoque linguíístico, que consiste en identificar y analizar los distintos nombres que reciben dichas inflorescencias así como proponer que los términos pacay(a) y chapay(a) no son de origen maya sino quechua, y que los términos vernáculos mayas son identificables por medio de su estructura fonológica y morfológica.

El objetivo principal de esta investigación es describir algunos aspectos referentes a la relevancia cultural del actual uso de las inflorescencias de estas dos pal-

\footnotetext{
${ }^{1}$ Los autores desean agradecer los valiosos comentarios de los dictaminadores que ayudaron a enriquecer este trabajo.
} 
mas. En segundo lugar y con base en argumentos principalmente paleoetnobotánicos y linguíśsticos, un segundo objetivo es proponer la hipótesis de que las inflorescencias de estas palmas fueron conocidas y usadas por los mayas durante el periodo Clásico Tardío (600 al 800 d.C. $)^{2}$ en las Tierras Bajas noroccidentales.

En la figura 1 se muestran dos fotos que corresponden a cada una de las palmas estudiadas en este trabajo, a saber, Astrocaryum mexicanum y Chamaedorea tepejilote:
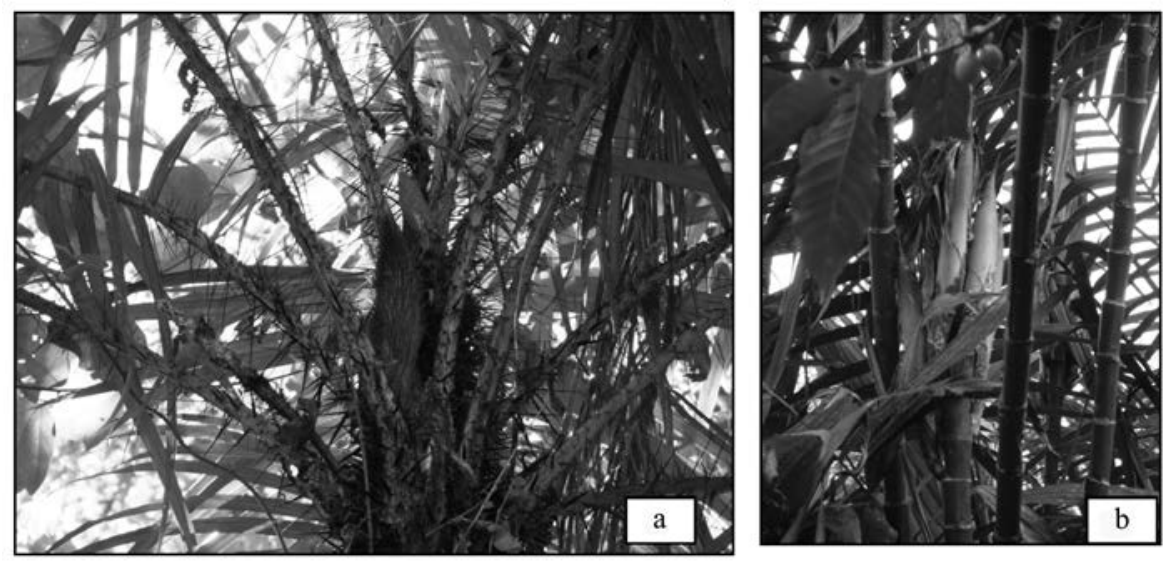

Figura 1. (a) Palma de chapay(a) (Astrocaryum mexicanum); (b) Palma de pacay(a) (Chamaedorea tepejilote). Fotografías de Adriana Caballero Roque.

El orden de presentación de los resultados que encontrará el lector es el siguiente: (1) en primer lugar se presenta la evidencia paleoetnobotánica y etnobotánica. (2) Posteriormente, en un segundo lugar mostramos los resultados del análisis nutrimental donde además se destacan algunos aspectos culturales del uso de ambos tipos de inflorescencias entre los tsotsiles de la región de la Biósfera "Selva El Ocote" así como en comunidades ch'oles de la región de Palenque. (3) Finalmente, se muestra el análisis linguiístico de los nombres de cada una de estas plantas.

\section{Evidencia paleoetnobotánica y etnobotánica}

Extracción de fitolitos a partir de sedimentos del basurero del palacio de Chinikihá

Durante la excavación llevada a cabo en la temporada de campo de enero a marzo de 2010, dentro del Proyecto arqueológico “Chinikihá: Un estudio arqueológico

\footnotetext{
${ }^{2}$ Los horizontes temporales varían de autor a autor, pero en este caso utilizamos las fronteras temporales citadas en Benavides Castillo (2001: 82).
} 
de un reino maya de la Cuenca del Usumacinta”, a cargo de Rodrigo Liendo, se recolectaron muestras de 20 gramos de sedimentos para la extracción de fitolitos en diferentes cuadros y profundidades de la cuadrícula de excavación del depósito del Palacio de Chinikihá (figura 2). Los fitolitos hallados en la muestra de sedimento fueron obtenidos a partir de los cuadros K4-L4, elemento 44, ubicado a una profundidad comprendida entre -10 y $-40 \mathrm{~cm}$ a nivel de superficie. El sedimento se encuentra en relación con un conjunto del complejo cerámico Murciélagos-Balunté (o complejo Ajín como se le atribuye actualmente a Chinikihá), característico del Clásico Tardío (650-800 d.C.).

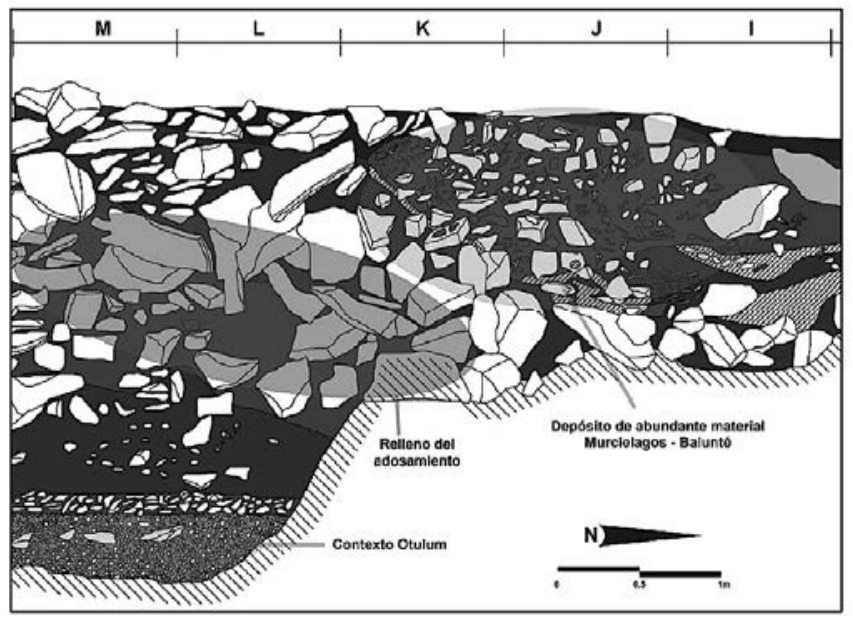

Figura 2. Ubicación en el perfil oeste de los contextos cerámicos Otulum y Murciélagos-Balunté del depósito problemático de la operación 114 palacio de Chinikihá, en relación al adosamiento al norte de la plataforma A-4h.

(Elaborado por Esteban Mirón 2012: 344; cortesía del Proyecto arqueológico "Chinikihá: Un estudio arqueológico de un reino maya de la Cuenca del Usumacinta”)

Posteriormente, se extrajeron fitolitos en el Laboratorio de Paleoetnobotánica y Paleoambiente del Instituto de Investigaciones Antropológicas con el apoyo de Cristina Adriano. La identificación de los fitolitos se realizó en el Laboratorio de Geología de la Universidad Nacional Autónoma de México bajo la supervisión de Alexandra Golyeva. Una vez que se observaron en microscopio, fue posible identificar la presencia de fitolitos de la familia ARECACEAE o PALMAE (figura 3).

Cabe mencionar que no fue posible identificar a nivel de género los fitolitos de palmas encontrados en el sedimento del depósito problemático del basurero del Palacio, sino que sólo se pudo identificarlos a nivel de familia ARECACEAE o PALMAE. En la región de Chiapas, se han reportado no menos de 13 géneros para la familia ARECACEAE, con los géneros siguientes: Acrocomia, Astrocaryum, Attalea, Bactris, Brahea, Chamaedorea, Cryosophila, Desmoncus, Geonoma, Paurotis, 


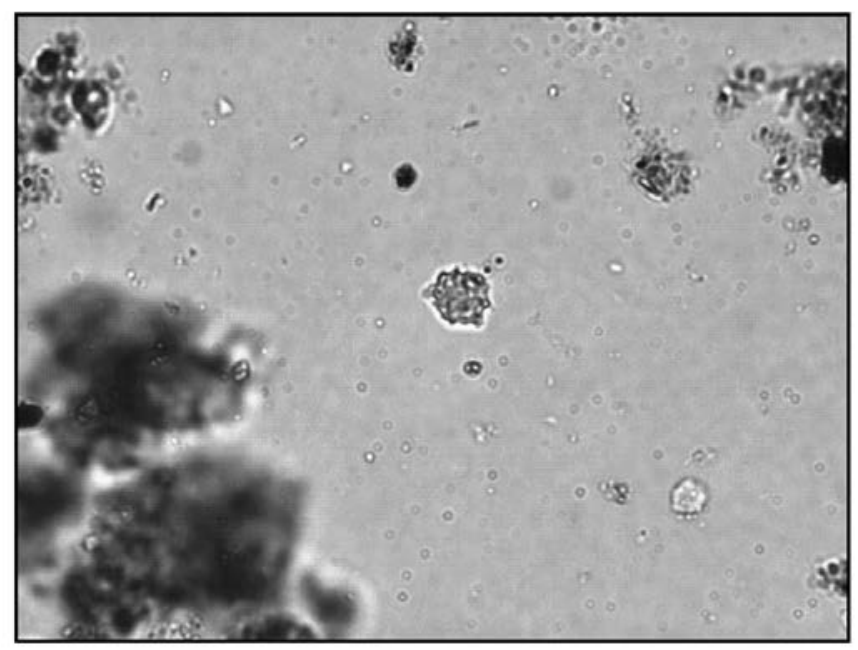

Figura 3. Fitolito tipo PALMAE extraído del sitio arqueológico Chinikihá (una forma general de fitolito pertenececiente a las palmas). Microfotografía: Alexandra Golyeva.

Reinhardtia, Sabal y Scheelea (Breedlove, 1986: 199). ¿A qué género de palmas podría corresponder el fitolito que aquí nos ocupa?

Cuando se obtiene algún fitolito de tipo PALMAE en un contexto arqueológico, tradicionalmente se le asocia a desechos de techos de palma de guano Sabal sp. (figura 4).

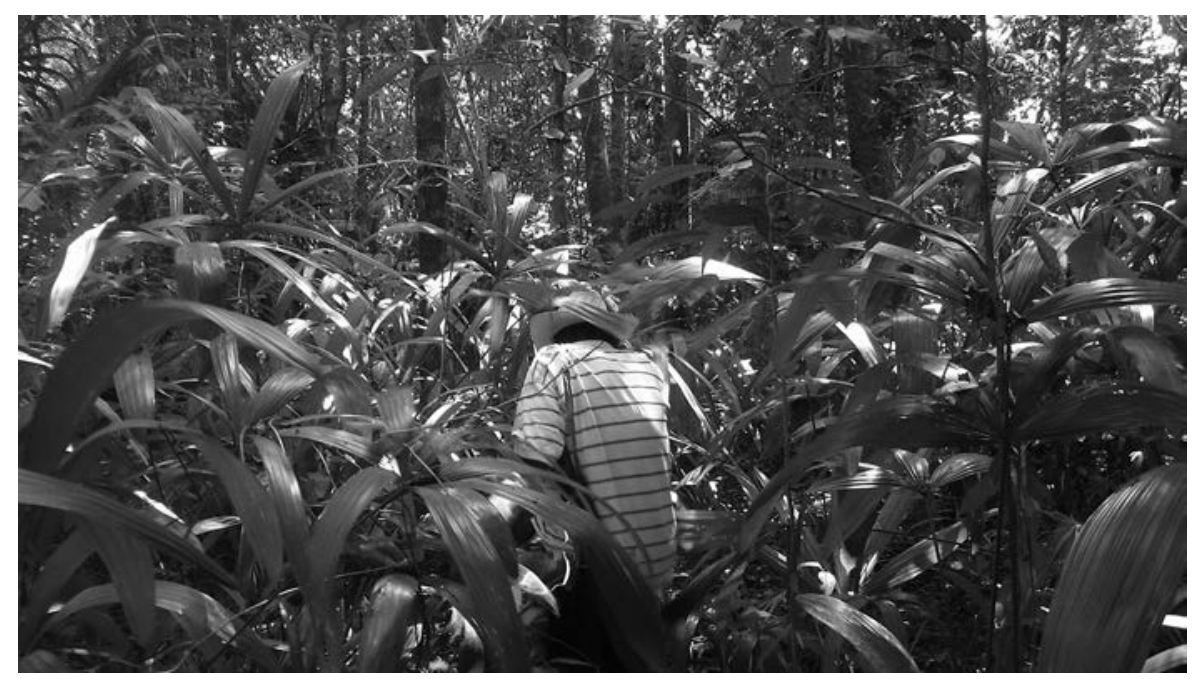

Figura 4. Guanal (Sabal sp.) en las planadas del sitio arqueológico Chinikihá, Chiapas, México. Foto Felipe Trabanino. 
Sin embargo, a partir de la singularidad del depósito (que por los elementos que contiene puede ser atribuido a los restos de un banquete), nosotros proponemos una hipótesis distinta que consiste en sugerir que el fitolito de tipo, PALMAE no necesariamente corresponde al género Sabal sp. (prototípicamente asociado a la construcción), sino que podría corresponder a alguno de los géneros de palmas comestibles. Los argumentos que apoyan la idea de que el depósito contiene los desechos comestibles de un banquete son de tipo arqueológico en tanto que, aunado a este fitolito tipo PALMAE, en el mismo depósito también se encontraron grandes cantidades de restos arqueozoológicos (muchos de ellos comestibles) como los xutes o caracoles de río (Pachychilus spp.), restos de venado cola blanca (Odocoileus virginianus) y más de 80 tiestos o tepalcates cerámicos de servicio tales como cajetes, ollas, cazuelas, platos, platos con glifos y cuencos (Mirón, 2012; Varela, 2012) (figura 5).

\section{Antecedentes arqueobotánicos de palmas en el área maya}

Diversas investigaciones realizadas en el área maya ${ }^{3}$ (Domenici, 2010; Lentz, 1990; McKillop, 1996; Piacenza, 2001) han reportado diferentes hallazgos de restos vegetales de palmas en dicha región desde el periodo Preclásico hasta el Posclásico. En particular, se habla de la presencia de restos vegetales de los géneros Acrocomia (coyol, cocoyol), Bactris (coconobo, pejibaye o chontaduro), Chamaedorea (xate), Cryosophila (escoba), Orbignya (corozo) y Sabal (guano, botán).

Por otro lado, se tiene evidencia de restos arqueobotánicos para el género Astrocaryum en Colombia, Brasil y Panamá, donde autores como Morcote-Ríos y Bernal (2001: 309) han sugerido que este género tuvo un uso posiblemente comestible desde el Holoceno tardío en estas regiones. Retomando la propuesta de Morcote-Ríos y Bernal (2001), pero en este caso en el área maya, sugerimos la hipótesis de que las inflorescencias de estas palmas tenían un uso comestible en la región de Chiapas durante el periodo Clásico y, siguiendo la metodología de Bronson (1966), nos apoyamos en evidencia etnobotánica, nutricional y linguíística para lanzar dicha hipótesis. Finalmente, las fuentes históricas también dan cuenta del uso de palmas en la alimentación en el área de nuestro estudio sugiriendo que ha habido un continuum en su uso desde la época prehispánica hasta la actualidad. Jan de Vos (2010: 82) cita: "Andaba fray Pedro ${ }^{4}$ siempre solitario por los montes, no llevando más tren que su breviario y un poco de pozol en una red, a usanza de los campesinos. Su dieta diaria consistía en hierbas y palmitos ${ }^{5}$ asados”. ¿De qué palmitos se alimentaba fray Pedro en la región palencana?

\footnotetext{
${ }^{3}$ El territorio conocido como "área maya" comprende los actuales estados de Tabasco, Chiapas, Yucatán, Campeche y Quintana Roo, en México, así como todo el territorio de Belice y Guatemala y parte de Honduras y de El Salvador.

${ }^{4}$ Fray Pedro Lorenzo de la Nada fue fundador de Palenque y, de acuerdo con De Vos, hablaba ch'ol, tseltal y tsotsil.

${ }^{5}$ Actualmente en Palenque se le conoce como 'palmito' a la flor de chapay(a) y no se le dice 'palmito' al corazón de la palma corozo del género Attalea.
} 


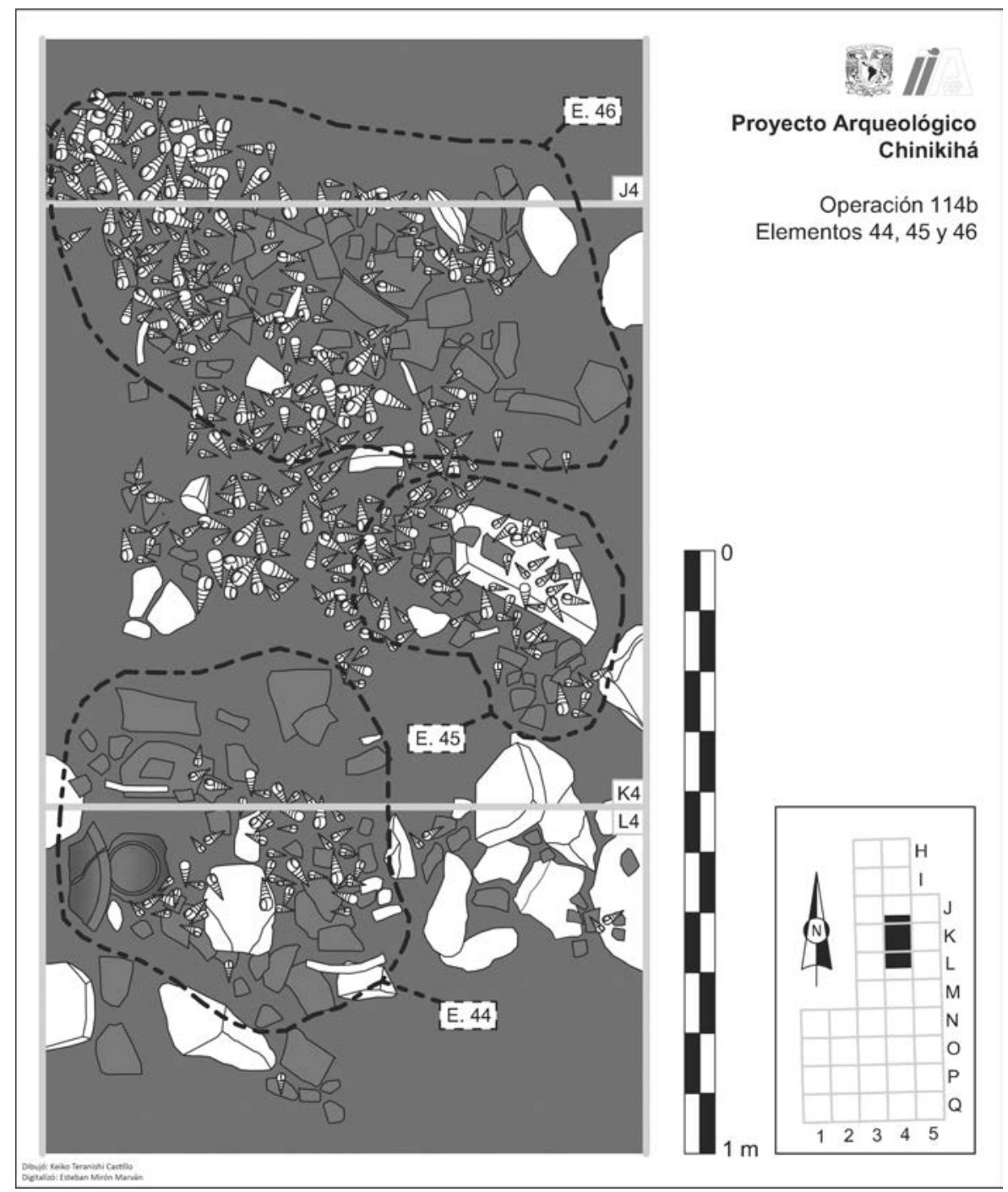

Figura 5. Vista en planta de los cuadros K4-L4 en donde fue hallado el fitolito de palma en conjunto al elemento no. 44 junto a huesos de venado cola blanca (Odocoileus virginianus), xutes (Pachychilus spp.) y cerámica utilitaria del período Murciélagos-Balunté.

(Dibujo de Keiko Teranishi Castillo, digitalización de Esteban Mirón; cortesía del Proyecto arqueológico "Chinikihá: Un estudio arqueológico de un reino maya de la Cuenca del Usumacinta”) 
A partir de la observación directa llevada a cabo durante dos temporadas de campo (enero a marzo de 2009 y enero a marzo de 2010), encontramos que en distintas regiones de Chiapas durante la época seca se consumen las inflorescencias inmaduras de las palmas de chapay(a) y de pacay(a). Se venden en los mercados y calles de Palenque, y se cocinan de muy diversas maneras (figura 6).

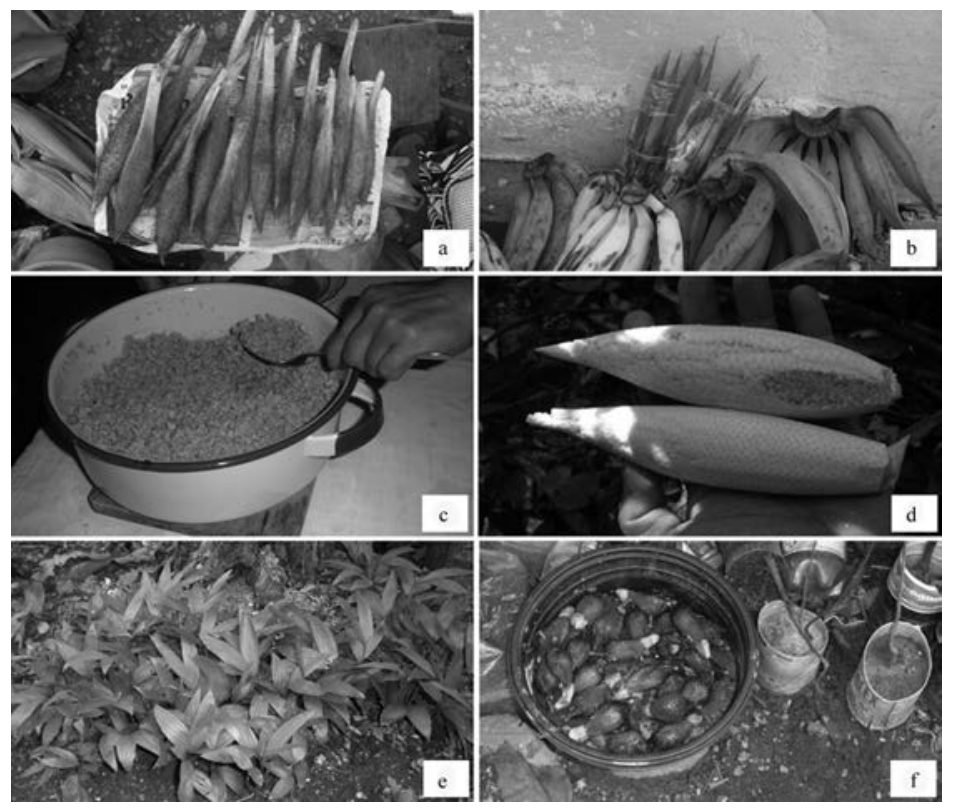

FiguRA 6. Venta de chapay(a) a tres por diez pesos mexicanos (a) y bolsa de "chibes" a diez pesos (b), preparación de chapay(a) para el almuerzo en el ejido de Reforma Agraria por Doña Canda y Doña Viola (c), cosecha de dos inflorescencias de chapay(a) del tamaño de dos manos en los cerros de Chinikihá en época seca $(d)$, retoños de pacayas en las cañadas de Chinikihá (e) y frutos de chapay(a) en germinación en el ejido de López Mateos (f) durante la época seca (marzo del año 2011) en los huertos familiares. Fotografías: Felipe Trabanino.

Dentro de los huertos familiares del ejido de López Mateos ${ }^{6}$ (véase mapa 1), se protegen los frutos del chapay(a) para trasplantarlos luego a los acahuales ${ }^{7}$ y enriquecer así las unidades del paisaje como sistema de manejo del bosque.

${ }^{6}$ Ejido aledaño al sitio arqueológico de Chinikihá.

7 "Acahual" es el nombre regional que recibe la milpa cuando ya no da maíz (también se conoce como guamil o rastrojo en otras regiones de Centroamérica). Se trata de una vegetación de tipo secundaria cuyo origen es a partir de la tala y del cultivo abandonado, después de lo cual se empieza a formar una sucesión secundaria que da origen al acahual. Pueden haber acahuales tanto de dos años de antigüiedad como de más de cincuenta años. 
También encontramos cultivos y cuidado de pacay(a) en las cañadas aledañas al sitio arqueológico de Chinikihá, y también cercanas al sitio de Palenque, donde se localizan asentamientos de grupos ch'oles. Bajo un sistema agroforestal, se cuidan a la sombra las plántulas de las palmas, en asociación con café (Coffea arabica), cacao (Theobroma cacao), y otros árboles útiles tales como el mango (Mangifera indica), el ramón (Brosimum alicastrum), el chicozapote (Manilkara zapota) y el cacaté (Oecopetalum mexicanum). Estas plántulas son transplantadas a otras áreas de actividad y cumplen además con la función de enriquecer los acahuales.

Por su parte, el uso actual de estas palmas comestibles nos permite sugerir que el conocimiento sobre las propiedades alimenticias data de épocas antiguas, ya que su uso con este fin está extendido con los ch'oles actuales, y también con tsotsiles y tseltales, por lo que presumiblemente se trata de un conocimiento heredado por estar compartido por distintos grupos que ocupan diferentes territorios. Además, y como mostraremos más adelante, los términos ch'olanos, tseltalanos y tsotsilanos asociados a ambas inflorescencias tienen un origen común, por lo que presumiblemente se trata de términos compartidos por grupos de lenguas que se escindieron desde hace mucho tiempo.

\section{Análisis del contenido nutricional}

En este apartado mostraremos el contenido de nutrientes de las dos inflorescencias perteneciente a las dos palmas que nos ocupan en este trabajo. Actualmente estas inflorescencias son usadas en la alimentación de la población tsotsil en la Reserva de la Biósfera "Selva El Ocote”, localizada en Chiapas, México, así como en las zonas aledañas al sitio arqueológico de Palenque, cuyos pobladores son ch'oles. La reserva "Selva El Ocote" es un área natural protegida rica en biodiversidad y en cultura etnobotánica, ya que en su zona de amortiguamiento las poblaciones tsotsiles conservan los conocimientos ancestrales mayas protegiendo, cultivando, domesticando y seleccionando recursos vegetales útiles para su alimentación. Este es el caso de las inflorescencias de las palmas Chamaedorea tepejilote y Astrocaryum mexicanum que se obtienen durante los meses de noviembre hasta abril (PMREBISO, 2000; Caballero Roque, 2010).

La metodología que se llevó a cabo para el análisis nutrimental de estas plantas fue de dos tipos: la primera fue la de observación directa aplicada con los ch'oles del ejido de López Mateos y el de Reforma Agraria durante dos temporadas de campo (de la cual ya se hicieron algunas anotaciones) y la otra fue a través de encuestas semiestructuradas ${ }^{8}$ a 25 mujeres de la comunidad Emiliano Zapata, en la reserva "Selva El Ocote" (véase mapa 1), donde se identificó a las

\footnotetext{
${ }^{8}$ De acuerdo con la metodología que se usa en etnobotánica, una encuesta de este tipo tiene una combinación de dos tipos de preguntas: unas cuya respuesta se espera que sea "abierta" y otras que restringen la respuesta, ya que se proporcionan opciones (una o dos) que el encuestado debe elegir como la respuesta correcta.
} 


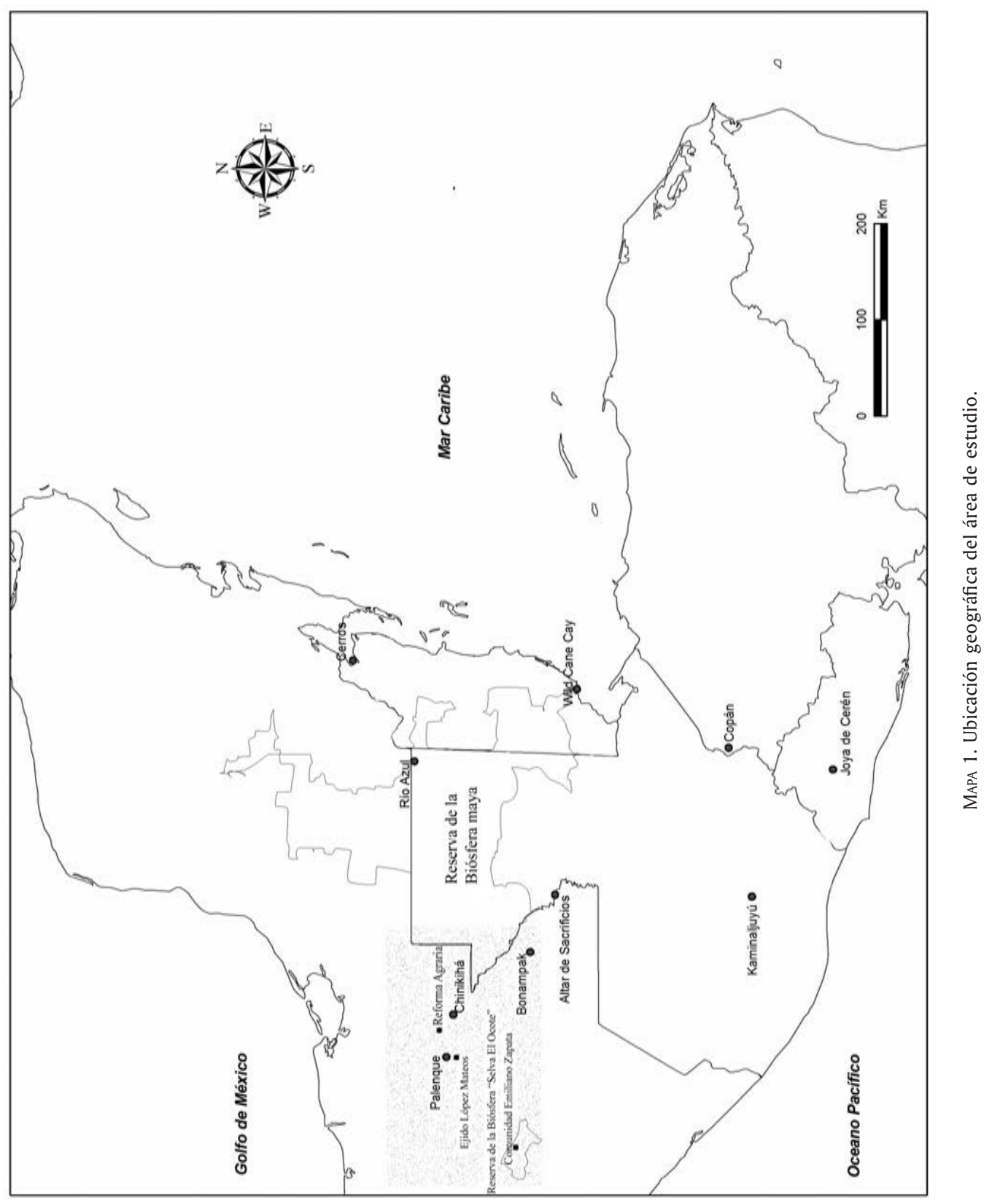


inflorescencias de chapay(a) y pacay(a) como vegetales comestibles utilizados en la estación seca.

El contenido de nutrimentos presentes en las inflorescencias se analizó en los laboratorios de Tecnología de Alimentos del Instituto Nacional de Ciencias Médicas y Nutrición Salvador Zubirán, en la ciudad de México. Se determinó humedad, cenizas, proteínas, grasas, hidratos de carbono, fibra y energía en 100 gramos de muestra, así como nutrimentos inorgánicos y vitaminas de acuerdo a las normas oficiales de México. Los resultados se presentan en base fresca ya que es la forma de uso entre la población.

Los datos para la comparación de valores nutricionales de maíz, frijol y calabaza se obtuvieron de tablas de valores nutritivos en base fresca (Ledesma et al., 2010).

La composición nutrimental de especies vegetales comestibles permite conocer la calidad de la dieta de la población que la consume y fomentar la conservación de este recurso natural para que continúe aportando beneficios para la alimentación. Sin embargo, se necesitan hacer otros análisis como el perfil de aminoácidos, además del análisis de otras especies vegetales de la misma zona que aún no han sido estudiadas.

\begin{tabular}{|c|c|c|c|c|c|}
\hline $\begin{array}{c}\text { Nombre } \\
\text { científico }\end{array}$ & $\begin{array}{c}\text { Nombre } \\
\text { común }\end{array}$ & $\begin{array}{c}\text { Energía } \\
\text { Kcal }\end{array}$ & $\begin{array}{c}\text { H de C } \\
\mathbf{g}\end{array}$ & $\begin{array}{c}\text { Proteína } \\
\mathbf{g}\end{array}$ & $\begin{array}{c}\text { Lípidos } \\
\mathbf{g}\end{array}$ \\
\hline $\begin{array}{c}\text { Astrocaryum } \\
\text { mexicanum Liebm. } \\
\text { exnMart. }\end{array}$ & chapay(a) & 50.8 & 7.6 & 4.2 & 0.4 \\
\hline $\begin{array}{c}\text { Chamaedora } \\
\text { tepejilote Liebm. }\end{array}$ & pacay(a) & 37.0 & 4.6 & 3.7 & 0.3 \\
\hline Zea mays L. & maíz & 365 & 74.26 & 9.42 & 4.74 \\
\hline $\begin{array}{c}\text { Phaseolus } \\
\text { vulgaris } L .\end{array}$ & frijol & 341 & 62.36 & 21.60 & 1.42 \\
\hline Cucurbita pepo & calabaza & 26 & 6.5 & 1.0 & 0.10 \\
\hline
\end{tabular}

TABLA 1. Comparación de cantidades de energía y macronutrientes de inflorescencias de palmas y otros vegetales, en $100 \mathrm{~g}$ de porción comestible.

\begin{tabular}{|c|c|c|c|c|c|c|c|}
\hline Nombre & $\begin{array}{c}\text { Calcio } \\
\mathbf{m g}\end{array}$ & $\begin{array}{c}\text { Fósforo } \\
\mathbf{m g}\end{array}$ & $\begin{array}{c}\text { Hierro } \\
\mathbf{m g}\end{array}$ & $\begin{array}{c}\text { Magnesio } \\
\mathbf{m g}\end{array}$ & $\begin{array}{c}\text { Sodio } \\
\mathbf{m g}\end{array}$ & $\begin{array}{c}\text { Potasio } \\
\mathbf{m g}\end{array}$ & $\begin{array}{c}\text { Zinc } \\
\mathbf{m g}\end{array}$ \\
\hline chapay(a) & 50.8 & 85.1 & 1.26 & 7.6 & 4.2 & 0.4 & 10.6 \\
\hline pacay(a) & 37.0 & 87.3 & 1.1 & 4.6 & 3.7 & 0.3 & 7.87 \\
\hline maíz & 158 & 235 & 2.71 & 147 & 1.0 & 284 & 2.21 \\
\hline frijol & 183 & 352 & 5.02 & 222 & 12 & 1042 & 3.65 \\
\hline calabaza & 19 & 41 & 0.8 & 21 & 3.0 & 281 & 0.32 \\
\hline
\end{tabular}

TABLA 2. Comparación de cantidades de minerales de palmas y otros vegetales, en $100 \mathrm{~g}$ de porción comestible. 


\begin{tabular}{|c|c|c|c|c|c|}
\hline Nombre & $\begin{array}{c}\text { Vit. A } \\
\mathbf{m g}\end{array}$ & $\begin{array}{c}\text { Ac. Asc. } \\
\mathbf{m g}\end{array}$ & $\begin{array}{c}\text { Tiamina } \\
\mathbf{m g}\end{array}$ & $\begin{array}{c}\text { Riboflav. } \\
\mathbf{m g}\end{array}$ & $\begin{array}{c}\text { Niacina } \\
\mathbf{m g}\end{array}$ \\
\hline chapay(a) & 0 & 0.25 & 0.002 & 0.26 & 1.28 \\
\hline pacay(a) & 0 & 0.24 & 0.032 & 0.16 & 1.56 \\
\hline maíz & 11 & 0.0 & 0.34 & 0.08 & 1.60 \\
\hline frijol & 0.0 & 0.0 & 0.63 & 0.17 & 1.80 \\
\hline calabaza & 369 & 9.0 & 0.06 & 0.04 & 0.50 \\
\hline
\end{tabular}

TABLA 3. Comparación de cantidades de vitaminas de palmas y otros vegetales, en $100 \mathrm{~g}$ de porción comestible.

Con todos los datos nutrimentales presentados en las tablas 1 a 3 se aprecia que las flores de las palmas analizadas en este trabajo tienen valores mayores del $50 \%$ de proteínas con respecto a las otras especies con las que se compararon, quedando muy por debajo de la aportación en proteínas con respecto al frijol.

En cuanto al contenido en minerales (tabla 2), nuevamente el frijol tiene un mayor contenido de calcio, hierro y zinc con respecto a las inflorescencias de la pacay(a) y de la chapay(a), pero si comparamos con la calabaza, estas últimas tienen mayor cantidad de minerales. De igual manera, y como se muestra en la tabla (3), las vitaminas riboflavina y niacina presentan una mayor concentración en las inflorescencias que aquí nos interesan con respecto a la calabaza. Centurión et al. (2009) reportan un estudio similar sobre contenido nutricional de inflorescencias de la zona de Tabasco, presentando los resultados en base seca.

En la zona de estudio ${ }^{9}$ se observa que en la actualidad el consumo de las inflorescencias de pacay(a) y chapay(a) combinadas con los alimentos básicos como maíz y frijol proveen de nutrimentos necesarios para una adecuada alimentación de la población. Cabe destacarse que el consumo de leguminosas es de gran importancia en la nutrición por el reducido consumo de alimentos de origen animal en la alimentación diaria de la población maya actual estudiada. De igual manera, al cosecharse en época de secas, los mayas prehispánicos pudieron haber usado estas inflorescencias como complemento o fuente principal de alimento entre las dos temporadas de cosecha de maíz.

Sobre el origen lingüístico de los nombres de las inflorescencias de $C$. tepejilote y de $A$. mexicanum.

Los términos pacay(a) y chapay(a) son usados en el sureste de México (Tabasco y Chiapas) para nombrar las inflorescencias comestibles de dos especies de

\footnotetext{
${ }^{9}$ El uso de la pacay(a) con fines comestibles ha sido atestiguado también en Oaxaca, Veracruz y Tabasco (Quero, 1992) dentro del territorio mexicano, así como en distintas regiones de Guatemala (Castillo, Gallardo y Johnson, 1994).
} 
palmeras (C. tepejilote y A. mexicanum) distribuidas geográficamente en distintas regiones de otros países tropicales como Belice, Guatemala, Costa Rica, Honduras, El Salvador, Panamá y Perú. Estos términos no son usados de manera unívoca para nombrar un solo referente, ya que el mismo término puede ser usado para nombrar otros referentes (como la Chamaedorea elegans, en el caso de pacay(a), por ejemplo). En el caso contrario a la homonimia y de manera más frecuente, encontramos variación entre los nombres comunes que dichas inflorescencias reciben en las distintas regiones donde se localizan.

En este apartado, proponemos que los términos pacay(a) y chapay(a) tienen un origen quechua. Como resultado del análisis, planteamos que pacay(a) entró como préstamo del quechua al español y, mostramos que el término vernáculo para pacay(a) en muchas lenguas mayas está registrado en diversos diccionarios como k'ib' o como čib' (según la lengua maya de la que se trate). ${ }^{10}$ Pacay(a) es la forma que se reconoce entre los hablantes de lenguas mayas como el nombre en español para č'ib' (en su forma ch'olana), por lo que en las lenguas mayas (y con base exclusivamente en los datos de los diccionarios) diríamos que el término vernáculo maya no alterna en los mismos contextos de uso con el término del español.

Por su parte, encontramos que chapay(a) es un préstamo introducido a las lenguas ch'olanas, tseltalanas y tsotsilanas (posiblemente desde el quechua, sin pasar por el español), mientras que los términos vernáculos mayas son ?ak te? en ch'ol y čitam te? en tseltal.

La distribución geográfica del término chapay(a) entre los hablantes de lenguas mayas parece estar mucho más limitada que el de pacay(a), ya que en contraste con este último término, chapay(a) aparentemente es reconocido sólo por hablantes de lenguas mayas de las Tierras Bajas (entre ellas ch'ol, tseltal y tsotsil) dado que el término no está registrado en todos los diccionarios consultados de lenguas mayas, como sí lo está pacay(a).

La lista de los distintos nombres encontrados hasta el momento para cada una de las palmas estudiadas se enlista en las tablas 4 y 5 :

\begin{tabular}{|c|c|c|}
\hline Nombre común & Región & Lengua de origen del término \\
\hline $\begin{array}{c}\text { chichón, chocho, guaya } \\
\text { de cerro, guaya de joma }\end{array}$ & Tabasco & no maya \\
\hline lancetilla & Honduras & \\
\hline chocón; chipi & Veracruz & \\
\hline güiscoyol & Oaxaca, Tabasco & \\
\hline cohune, warren cohune & Belice (belice creol) & \\
\hline
\end{tabular}

${ }^{10}$ El fonema *k del protomaya tiene distintos reflejos en las lenguas mayas hijas. En las lenguas ch'olanas ocurrió un proceso en donde * ${ }^{*}>\check{c}$, mientras que en otras lenguas como las yucatecanas, * $\mathrm{k}>\mathrm{k}$. 


\begin{tabular}{|c|c|c|}
\hline tzitzún & San Fernando Chiapas (zoque) & \\
\hline chapay, chapaya & Tabasco y Chiapas & maya \\
\hline Pak te?, chapıy & $\begin{array}{c}\text { Tumbalá, Tila y Sabanilla } \\
\text { (ch’ol) }\end{array}$ & \\
\hline čitam te?, čapay te? & tseltal & \\
\hline
\end{tabular}

TABLA 4. Nombres comunes para Astrocaryum mexicanum.

Los datos mostrados en las tablas 4 y 5 están organizados en dos grupos: los nombres que tienen un origen no maya (como caña verde, palmito dulce, lancetilla, chicuilote, tepejilote, nuru, cohun, güiscoyol, etc.) y los que tienen origen maya. Recordando que el objetivo central de este trabajo es entender la relación de estas palmeras con grupos mayas actuales (y también posiblemente prehispánicos) de la región de las Tierras Bajas mayas, nos centraremos en la discusión de los términos con los que se nombran a estas dos especies de palmeras en las lenguas mayas de dicha región ${ }^{11}$ y mostraremos los argumentos que sostienen que los términos pacay(a) y chapay(a) no tienen un origen maya. Finalmente, presentaremos la propuesta de cuáles son los términos propios de estas lenguas con base en argumentos de tipo estructural y de linguística comparativa.

\begin{tabular}{|c|c|c|}
\hline Nombre común & Región & $\begin{array}{c}\text { Lengua de origen } \\
\text { del término }\end{array}$ \\
\hline pacay(a) & $\begin{array}{c}\text { Costa Rica, Guatemala, } \\
\text { Honduras, Colombia }\end{array}$ & No maya \\
\hline tepejilote & Oaxaca & \\
\hline gueecho guiaroo & Oaxaca & \\
\hline yetzo-yaa & Guatemala & \\
\hline chicuilote, chimp, capuca & Costa Rica & \\
\hline juyuk; koo boo & Panamá & \\
\hline Nuru & Costa Rica & maya \\
\hline guarrazú & Panamá & \\
\hline Caña verde & Alta Verapaz, Guatemala & \\
\hline palmito dulce & Chiapas & \\
\hline bojón & & \\
\hline chib' [či & Quetzaltenango, Guatemala & \\
\hline
\end{tabular}

TABLA 5. Nombres comunes para Chamaedorea tepejilote.

${ }^{11}$ De acuerdo con Centurión et al. (2009: 194). 
El primer argumento que nos permite proponer que chapay(a) y pacay(a) no son raíces mayas es principalmente estructural, ya que aun cuando los fonemas de tales términos forman parte del inventario fonológico de las lenguas mayas, estos tienen una estructura silábica CVCVC(V) que no es típica de estas lenguas, lo que los hace estructuralmente "sospechosos".

Estamos conscientes de que, aunque la gran mayoría de las raíces mayas tienen una estructura de templete silábico CVC, también existen raíces mayas de tipo CVCVC. Sin embargo, éstas pueden ser consideradas excepciones, como señala Coon (2004b: 71):

Exceptions from the CVC roots certanly exist. Frecuently-used nouns such as winik 'man' 'ixik 'woman', and 'ixim 'corn', for example do not conform to the canonical CVC root pattern. It is likely, however, that they have been created from morphological processes (note the identical -ik endings of the first two) wich are no longer poductive. Certanly, it is safe to say that CVC roots form the base of Mayan languages.

Es decir, que las formas tanto sincrónicas actuales como aquellas atestiguadas en la colonia con una estructura no-CVC deben ser vistas como formas derivadas morfológicamente (posiblemente en algún momento temprano de su historia), y que es en el eje del análisis diacrónico donde se podría esclarecer el origen de los morfemas que la componen. Como señala la misma Coon (ibídem), no es casual tampoco que muchas veces la vocal de la segunda sílaba sea armónica con la primera $\mathrm{CV}_{1} \mathrm{CV}_{1} \mathrm{C}$ (como en los mismos ejemplos citados en el párrafo anterior), dado que se trata de procesos de armonía vocálica especialmente de los sufijos -VC con respecto a la raíz.

La postura de que las lenguas mayas tienen una estructura silábica predominantemente CVC es compartida por autores como England (2001); Lois y Vapnarsky (2001); Coon (2004a y b) Kaufman y Justeson (2003), entre muchos otros mayistas. Las raíces que actualmente tienen una estructura no-CVC entran dentro de lo que Coon llama "excepciones" y pueden ser analizadas como formas compuestas de dos o más morfemas que en algún momento de su historia se lexicalizaron en un solo morfema - ahora ya no analizable-, donde el sufijo que alguna vez fue divisible perdió productividad, y con ello su significado, pasando a formar parte de una raíz, conservando su contenido fónico pero no semántico.

Ahora bien, si quisiéramos sostener que pacay(a) y chapay(a) forman parte de las raíces mayas "excepcionales", esta idea se derrumba ante la evidencia comparativa con la presencia de cognadas en muchas lenguas mayas (en particular del término para pacay $(a)^{12}$ ) de términos claramente de origen maya que cumplen con la estructura de templete silábico CVC. Por su parte, los reflejos en las len-

\footnotetext{
${ }^{12}$ Véase el MED de Kaufman y Justeson (2003: 1118), o tabla 6 de este trabajo.
} 
guas mayas para el término para chapay(a) no es tan regular como pacay(a), sin embargo, y como mostraremos con detalle, también están atestiguadas raíces con forma $\mathrm{CVC}$, que son las que nos permiten rastrear y proponer cuál es el término vernáculo maya.

El tercer argumento que nos permite proponer que se trata de términos cuyo origen es quechua es que ambos lexemas están presentes en diversos diccionarios de esta lengua aunque, como veremos, ha habido un desplazamiento de significado, ya que el referente en ninguno de los dos casos es el mismo en quechua que en lenguas mayas. Finalmente, en el Diccionario de la Real Academia de la Lengua Española (DRAE), pacay(a) se reconoce como un término de origen quechua.

\section{El término chapay(a)}

El término chapay(a) puede ser usado para referirse a la palma completa, o bien, sólo a la inflorescencia, ya que en algunos casos se explica que "chapay(a) es la flor de la palma llamada chichón". ${ }^{13}$ En tanto que muchos de los datos linguiísticos fueron extraídos de vocabularios así como de investigaciones cuyo fin no era la descripción y/o análisis linguiístico, ${ }^{14}$ estamos conscientes de que se requeriría de un trabajo linguiístico sistemático en investigación de campo para determinar si en la actualidad los ch'oles, los tsotsiles y los tseltales hacen esta diferencia explícita en su lengua. Debido a que la mayoría de los datos no fueron recolectados por nosotros, no podemos asegurar que en todos los casos se haga esta diferencia y, por esta razón, hemos decidido enlistar ambos referentes (la inflorescencia y la palma completa) bajo el mismo nombre.

Según los datos registrados en los diccionarios de López K'ana et. al. (2005) y de Hofling y Fernando Tesucún (2000), en algunas variantes de ch'ol, tojolabal, tseltal y tsotsil actuales, el término chapay(a) se reconoce como el término maya (que nosotros señalamos como préstamo del quechua) para nombrar a la palma de Astrocryum mexicanum. Llama la atención que en estos diccionarios no se registró el término vernáculo maya, ya que esto podría indicar que en algunas variantes de estas lenguas el préstamo ha desplazado de manera definitiva a la forma maya original. Sin embargo, también es de notarse que el desplazamiento del término vernáculo por el préstamo no ha ocurrido en todas las variantes de lenguas mayas de las Tierras Bajas y, en todo caso, lo que encontramos es que la forma vernácula alterna en el discurso con la forma préstamo, pero los hablantes distinguen ambas formas. Esto se puede constatar en el diccionario de ch'ol (Aulie y Aulie (1996[1978]), donde se

${ }^{13}$ Esto también ocurre para pacay(a) (como mostraremos con detalle más adelante, en la tabla 6), donde el término también puede referir a una parte de la palma, a toda la palma, e incluso posiblemente a alguna palma distinta de la $C$. tepejilote, que aquí nos ocupa.

${ }^{14}$ Como el trabajo de Sanders (2011), cuyo fin es el de hacer un catálogo a manera de herbario en el que se recolectaron datos de los distintos nombres que reciben las dos palmeras en cuestión y que son citados en este trabajo. 
especifica que la forma chapay(a) es el nombre en español para la flor de palmera ?ak te?.$^{15}$ Por su parte, en tseltal hay dos formas registradas: chapay(a) te? y čitam te? (Berlin et al. 1974). La forma chapay(a) te? se explica como el término adaptado a las reglas morfológicas del idioma tseltal. La adaptación fonológica del término también ocurrió en algunas variantes de ch'ol donde el término chapay(a) se realiza como čapıy, con la sexta vocal en la segunda sílaba, lo que nos indica que es un préstamo ya adaptado a la fonología de la lengua receptora - por lo que ya forma parte del inventario léxico de la lengua-. Por su parte, el nombre čitam te? está compuesto por la palabra para čitam 'puerco' en tseltal, así que la traducción podría ser 'palo puerco', donde posiblemente recibe este nombre en tseltal por analogía con las espinas del cochemonte o puercoespín. Ahora bien, las formas chapay(a) te?, čitam te? y ?ak te? comparten la raíz te?, que significa 'árbol', 'palo', 'madera' en todas las lenguas mayas. Bajo la idea de que chapay(a) es un préstamo (asimilado a la lengua receptora en algunos casos), nos quedan dos posibles candidatos para nombrar a esta inflorescencia como la forma maya original de las Tierras Bajas: čitam y ?ak. Desafortunadamente, la poca evidencia linguíística sobre el nombre de esta planta registrada en los diccionarios y gramáticas consultadas no da la posibilidad de elegir de manera definitiva entre ambos nombres, por lo que por el momento proponemos que en tseltal el nombre maya es čitam te?, mientras que en ch'ol es ?ak te?, con una estructura silábica CVC, prototípica de las raíces mayas, donde el cierre glotal inicial tiene un valor consonántico.

\section{El término pacay(a)}

De acuerdo con los datos presentados por Kaufman y Justeson (2003: 1118), el término protomaya * $k$ 'iib' 'palma de pacaya' tiene reflejos transparentes en lenguas mayas de las Tierras Altas y de las Tierras Bajas. La forma se realiza como čib' en lenguas ch'olanas y tseltalanas y fue reconstruida por los autores en protoch'olano como *čib', como se muestra en la tabla 6.

\begin{tabular}{|l|l|l|}
\hline yucateco colonial & ch'ib' & arista/punta de palma, etc. \\
\hline Itzaj & ch'ib' & (flor de) pacay(a), tepejilote \\
\hline mopán & ch'ib' & pacay(a) \\
\hline protoch'olano & 'ch'ib' & palma de pacay(a) \\
\hline ch'ol & ch'ib' & palma que da fruta comestible \\
\hline tsotsil & ch'ib' & palmita \\
\hline tseltal & ch'ib' & palma (para adornos) \\
\hline Chuj & k'ib' & pacay(a): palma para adornar \\
\hline
\end{tabular}

${ }^{15}$ Otro de los datos que apoyan que la forma vernácula maya es $P a k$ te? es que, de acuerdo con Justeson et al. (1985: 10), el kekchí tomó como préstamo del ch'ol el término ?ak te? para el fruto de esta palma. 


\begin{tabular}{|l|l|l|}
\hline poptí & ch'ib' & pacay(a) \\
\hline kanjobal & ch'ib' & palma (fénix) \\
\hline kiché & k'iib' & $\begin{array}{l}\text { planta silvestre parecida a la } \\
\text { pacay(a), se usa la hoja como } \\
\text { adorno }\end{array}$ \\
\hline tzutujil & k'iib' & $\begin{array}{l}\text { pacay(a) de monte (chamaedo- } \\
\text { rea sp.) }\end{array}$ \\
\hline pocomchi & k'iib' & pacay(a) \\
\hline kekchi & k'ib' & pacay(a) \\
\hline
\end{tabular}

TABLA 6. Nombres mayas para pacay(a) según Kaufman y Justeson (2003: 1118).

En esta tabla, se muestra que el significado en cada una de las lenguas mayas puede aludir a más de un referente: ya sea a alguna parte de la palma (como "arista de", "punta de" o "flor de") o bien a "alguna planta silvestre parecida a la pacaya”. En otros casos, se habla de 'una palma para adornar', sin mayor detalle en la descripción, lo que deja abierta la posibilidad de que el referente en algunas lenguas mayas pudiera ser distinto del de la Chamaedorea tepejilote. Un estudio etnobotánico profundo en cada una de las lenguas mayas nos ayudaría a esclarecer si el referente es el mismo en cada una de estas lenguas, o bien si se conserva la forma cognada pero con un desplazamiento de significado con alusión a un referente distinto a la especie que estamos estudiando. El hecho que nos interesa enfatizar es que al menos en ch'ol actual de la región de Palenque, el término vernáculo maya con el significado heredado desde el protomaya sí tiene el referente de la $C$. tepejilote, y este dato está constatado tanto por nosotros a partir del trabajo de campo, como por los propios datos de Kaufman y Justeson (ibídem), quienes en su entrada léxica de ch'ol lo definen como 'palma que da fruta comestible', y en la de tzutujil, como 'pacaya de monte (Chamaedorea sp.)'.

Por otro lado, y apoyando la hipótesis presentada acerca del uso prehispánico de las inflorescencias de estas palmas, la evidencia linguiística sugiere que la presencia del término *k'iib' del protomaya (Kaufman y Justeson, 2003: 1118) con claras cognadas en todas las lenguas mayas tiene una profundidad temporal importante y que, al estar presente en la mayoría de ellas, dicha planta se conocía desde épocas tempranas por estos grupos.

Considerando la evidencia presentada en la tabla 6, podemos afirmar con certeza que el término č'ib' es el nombre ch'olano y tseltalano para 'la flor de pacay(a)', ya que cumple con la estructura fonológica de las raíces mayas CVC, además tener cognados en buena parte de las lenguas de la familia linguiística maya (como se constata en el MED).

El origen quechua de pacay(a) y chapay(a)

De acuerdo con Landerman (1973: 74), pacay es el nombre quechua que recibe la fruta de la leguminosa "paterno" Inga edulis, mientras que dos informantes 
peruanos (c.p.) nos indicaron que pacay es un vocablo de uso común en la costa sur de Perú para nombrar a una "vaina con fruto". El término está atestiguado en el DRAE como "pacay. (Del quechua páqay) 1. m. Am. Mer. guamo (Il árbol mimosáceo). 2. m. Am. Mer. Fruto de este árbol” y, como "pacaya. 1. f. Am. Cen. Variedad de palmera de hojas pinnadas. Las yemas de sus flores son comestibles, al igual que los cogollos y tallos delgados y tiernos. 2. f. Guat. Tarea enojosa”. También está atestiguado en el Corpus Diacrónico del Español (CORDE) en un documento fechado en 1595-1615 de Perú, en un contexto donde se enlistan frutos de plantas ${ }^{16}$ y también en otro de $1877 .{ }^{17}$

¿Pero cómo pudieron haber llegado estos términos quechuas al vocabulario de los hablantes de lenguas ch'olanas y tseltalanas? Durante la colonización de Chiapas, entre los años 1528 y 1548, Diego de Mazariegos realizaba actividades de intercambio de esclavos desde México (en particular desde Chiapas) hacia las Antillas y Perú (De Vos, 2010: 34) y posiblemente, en ese ir y venir, trajo consigo algunos vocablos que más tarde los españoles utilizarían para nombrar a referentes similares. Tal parece ser el caso del nombre pacay(a) para nombrar a la flor de la $C$. tepejilote por analogía, ya que esta última también tiene forma de vaina, en tanto que, como ya mencionamos, pacay(a) no tiene el mismo referente en México que en Perú.

Ahora bien, chapay(a) es un término quechua que no guarda ninguna relación semántica —aunque evidentemente sí formal— con la inflorescencia que recibe el mismo nombre en español de la región sureste de México. Llama la atención que el término chapay(a) no está registrado en el DRAE ni tampoco en el CORDE, pero que sí está atestiguado en la época colonial en distintos documentos de Perú referentes a cuestiones de tipo agrario. De acuerdo con Dedenbach-Salazar (1990: 149), en la Colonia, chapay(a) fue usado como "sinónimo de unancha-, tienen el siguiente significado: 'apoderarse apropiarse cosa baldía común y señalarla para sí'... [ ] se refiere a la repartición de tierras”.

Por su parte, y relacionada semánticamente con lo que explica DedenbachSalazar (1999: 207), la palabra chapay(a) significa 'jurisdicción', también atestiguado en la época colonial. De acuerdo con Gruszczynska (1995: 81), el "chapay(a) guanlo" es un tono referente a la música andina, registrado también en documentos coloniales. Un uso más reciente del término fue reportado por Carranza Romero (1978: 87), quien señaló que tsapay o chapay(a) significa 'atrapar' , 'atajar' y, en el quechua de Bolivia está registrado por Laime Ajacopa et al. (1997) como "chapatiyay, chapay, qaymiway, qhawmiy, watiqay. tr. 'Acechar'. 'Observar cautelosamente con algún propósito'. 'Espiar'. chapay. tr. Tapar acequia’ (Pot.)”.

\footnotetext{
${ }^{16}$ Retomado del CORDE: “* pinos, cachum, *plántanos, uayauas, sauindo, pacay, *uauas, lucuma, *paltas, *usum, *cirgüielas ***” (Felipe Guamán Poma de Ayala, El primer nueva crónica y buen gobierno, p. 64).

17 Retomado del CORDE: "ieron la empresa, logrando descubrir los palos de pacay, esteras de caña y el loro. Al encontrarse con ${ }^{*}$ " (Ricardo Palma, Tradiciones peruanas, p. 385.)
} 
Los resultados de los datos encontrados hasta ahora exigirían realizar una búsqueda más exhaustiva en documentos coloniales del español peruano para rastrear si el término pacay(a) está atestiguado como préstamo del quechua en otros documentos además de los que están en el CORDE. En cuanto al término chapay(a), vemos que está registrado en documentos coloniales, y que está reconocido como quechua tanto en dichos documentos como en distintos diccionarios de esta lengua (tanto de Perú como de Bolivia), pero resalta el hecho de no estar registrado ni en el español actual (en el DRAE) ni atestiguado en los registros del español peruano colonial del CORDE.

Por otro lado, y en el campo del significado, la introducción del préstamo pacay(a) al español se explica por una posible analogía en la forma de los frutos, ya que el referente en Perú y el referente en el área maya estudiada comparten la característica de tener forma de vaina. Sin embargo, la presencia del término chapay(a) en lenguas mayas resulta más difícil de rastrear y explicar, ya que los significados encontrados tanto en la Colonia como en la época actual refieren a verbos ("apoderarse de tierras", "acechar", "atrapar", "tapar acequia", etc.), o bien a un término de la música andina, pero nada relacionado con el referente que se encuentra en la actualidad en nuestra zona de estudio.

\section{Conclusión y discusión}

Siguiendo la tradición de otros estudios como el de Bronson (1966), en este trabajo buscamos conjuntar tres tipos de análisis en torno al uso comestible de las inflorescencias de las palmas pacay(a) y chapay(a) en la región de Chiapas, México. Partiendo de evidencia arqueológica (presencia de un fitolito tipo PALMAE en Chinikihá, Chiapas), histórica (referencia de fray Pedro de la Nada sobre el consumo de "palmitos" por los ch'oles de Palenque durante la Colonia), etnográfica actual (uso comestible de estas plantas entre ch'oles, tsotsiles y tseltales) y linguística (presencia de cognadas de al menos uno de estos términos en la mayoría de las lenguas mayas), sugerimos que el conocimiento y uso de dichas inflorescencias por grupos mayas de las Tierras Bajas con fines comestibles se remonta hasta épocas prehispánicas.

El análisis etnobotánico muestra la relevancia cultural que tienen estas flores entre algunos grupos mayas actuales, y el análisis nutrimental nos permite conocer cuál es el aporte nutricional a la dieta de dichos grupos. Por su parte, con el análisis linguiístico mostramos que el nombre ch'olano čib' ("k'iib' del protomaya), presente en todas las lenguas mayanses, y los nombres ?ak te? o čitam te? en las lenguas ch'olanas y tseltalanas respectivamente, son los vocablos en las lenguas vernáculas para denominar a estas inflorescencias.

La presencia de estos términos en la mayoría de las lenguas mayas evidencia que los mayas prehispánicos ya conocían, clasificaban y denominaban dichas plantas. 
Finalmente, y como dato marginal de esta investigación, también señalamos que existen dos volcanes que tienen una clara referencia a las palmas que aquí estudiamos: uno en Chiapas, el Chichonal, y otro en Guatemala, el Pacaya. Sería interesante seguir con este tipo de investigaciones pluridisciplinarias realizando colecciones de referencia con énfasis arqueológico para poder identificar fitolitos de palmas más allá de la familia, y también análisis de antropología física, en los que se puedan identificar aminoácidos específicos asociados a las flores de estas palmas. La propuesta atrevida de esta investigación invita, sin embargo, a cruzar resultados de estudios de contenidos nutrimentales con análisis linguiústicos, con el fin de proponer nuevas hipótesis en cuanto al uso de palmas en la subsistencia maya.

\section{BIBLIOGRAFÍA}

Aulie, H. Wilbur y Evelyn W. Aulie (comps.)

1996 [1978] Diccionario Ch'ol de Tumbalá, Chiapas, con variaciones dialectales de Tila y Sabanilla. Reeditado por Emily F. Stairs. México: Instituto Linguístico de Verano. (Serie de Vocabularios y Diccionarios Indígenas Mariano Silva y Aceves, 121). Disponible en: <www.sil.org/mexico/maya/chol-tumbala/S121aDiccionario-CTU.htm>.

Benavides Castillo, Antonio

2001 [1995]."El sur y el centro de la zona maya en el Clásico”, Historia Antigua de México, volumen II: El horizonte Clásico, Linda Manzanilla y Leonardo López Luján (eds.). México: Instituto Nacional de Antropología e Historia, 79-118.

Berlin, B., D. E. Breedlove y P. H. Raven

1974 Principles of Tzeltal Plant Classification. New York / London: Academic Press.

Breedlove, Dennis E.

1986 Listados florísticos de México, IV. Flora de Chiapas. México: Universidad Nacional Autónoma de México, Instituto de Biología.

Bronson, Bennet

1966 "Roots and the Subsistence of the ancient Maya", Southwestern Journal of Anthropology, 22: 251-279. Albuquerque: University of New Mexico.

Caballero Roque, Adriana

2010 Plantas comestibles no cultivadas de la Reserva de la biosfera Selva el Ocote. México: Universidad de Ciencias y Artes de Chiapas.

Carranza Romero, Francisco Javier

1978 La vigencia del kechwa en el Perú. Trujillo: Proyección Social de la Universidad Nacional de Trujillo. 
Castillo, M., N. Gallardo y D. Johnson

1994 "The pacaya palm (Chamaedorea tepejilote, Arecaceae) and its food use in Guatemala”, Economic Botany, 48(1): 68-75. New York: The New York Botanical Garden Press.

Centurión-Hidalgo, Dora, M. J. Alor-Chávez, J. Espinosa-Moreno, E. Gómez-García, M. L. Solano y J. E. Poot-Matu

2009 "Contenido nutricional de inflorescencias de palmas en la sierra del estado de Tabasco". Disponible en: < http://redalyc.uaemex.mx/src/inicio/ArtPdfRed. jsp?iCve $=15416281001>$.

Coon, Jessica

2004a. "Nominal constructions and split-ergativity in Chol Mayan. Proceedings of the 30th Meeting of the Berkeley Linguistics Society”, BLS, 30: 34-45, Marc Ettlinger, Nicholas Fleischer, and Mischa Park-Doob (eds.). Berkeley: Berkeley Linguistics Society.

Coon, Jessica

2004b. "Roots and Words in Chol (Mayan): a Distributed Morphology Approach". Bachelord Thesis. Reed College: Portland.

Dedenbach-Salazar Sáenz, Sabine

1990 Inka pachaq llamanpa willaynin - Uso y crianza de los camélidos en la época incaica. Estudio lingüístico y etnohistórico basado en las fuentes lexicográficas y textuales del primer siglo después de la conquista. Bonn: BAs.

Domenici, Davide

2010. "Patrones de uso ritual del espacio hipogeo en la selva El Ocote (Chiapas)", VI Coloquio Pedro Bosch Gimpera. Lugar, espacio y paisaje en arqueología: Mesoamérica y otras áreas culturales, Edith Ortiz Díaz (ed.). México: Universidad Nacional Autónoma de México, Instituto de Investigaciones Antropológicas, 349-386.

De Vos, Jan

2010 Fray Pedro Lorenzo de la Nada. Misionero de Chiapas y Tabasco. México: Fondo de Cultura Económica / Centro Interdisciplinario de Estudios Superiores de Antropología Social (Colección Centzontle).

England, Nora

2001. Introducción a la gramática de los idiomas mayas. Guatemala: PLFM / CHOLSAMAJ.

Gruszczynska, Anna

1995 El poder del sonido: el papel de las crónicas españolas en la etnomusicología andina. Cayambe, Ecuador: Biblioteca Abya-Yala.

Hofling, Charles Andrew y Félix Fernando Tesucún

2000 Tojt'an: Diccionario Maya Itzaj-Castellano. Guatemala: CHOLSAMAJ. 
Justeson, John, William Norman, Lyle Campbell, y Terrence Kaufman

1985 The Foreign Impact on Lowland Mayan Language and Script. Tulane: Middle American Research Institute (Publication 53).

Kaufman, Terrence y John Justeson

2003 A Preliminary Mayan Etymological Dictionary. Disponible en: < http://www.famsi.org/reports/01051/index.html>.

Laime Ajacopa, Efraín Cazazola, Félix Layme Pairumani y Pedro Plaza Martínez

1997 Diccionario Bilingüe quechua-castellano, castellano-quechua. La Paz: Centro cultural JAYMA. Disponible en: <www.illa-a.org/cd/diccionarios/DicQuechuaBolivia.doc>.

Landerman, Peter

1973 Vocabulario del quechua del Pastaza. Yarinacocha, Perú: Instituto Linguíístico de Verano / Ministerio de Educación (Serie Linguíística Peruana, 8).

Lentz, David

1990 "Acrocombia mexicana: Palm of the Ancient Mesoamerican", Journal of Ethnobiology, 10 (2): 183-194. Boston: University of Massachusetts.

Ledesma, José, Adolfo Chavez, Fernando Pérez, Eduardo Mendoza, Concepción Calvo y Miriam Muñoz de Chavez

2010 Composición de alimentos. Valor nutritivo de los alimentos de mayor consumo. México: Mc Graw Hill.

Lois, Ximena y Valentina Vapnarsky

2001. Polyvalence of Root Classes in Yucatekan Mayan Languages. Munchen: LINCOM Academic Publishers (LINCOM Studies in Native American Linguistics, 47).

López K’ana, Josías, Miguel Sántiz Méndez, Bernabe Montejo, Pablo Gómez Jiménez, Pablo González Casanova.

2005 Diccionario multilingüe = svunal bats'i k'opetik: español, tseltal, tsotsil, ch'ol, tojol-ab'al de Chiapas. México: Siglo XXI.

McKillop, Heather

1996 "Prehistoric Maya Use of Native Palms: Archaeobotanical and Ethnobotanical Evidence", The Managed Mosaic: Ancient Maya Agriculture and Resource Use, S. Fedick (ed.). Utah: University of Utah Press, 278-294.

Mirón, Esteban

2012 "Anexo 1, Análisis del material cerámico de la Operación 114 ”, Informe, cuarta temporada, Proyecto Arqueológico Chinikihá, 2011. Report submitted to the Instituto Nacional de Antropología e Historia, México, ANEXO 1, Rodrigo Liendo (ed.), 340-363. Disponible en: <http://www.mesoweb.com/ resources/informes/Chinikiha2011.html > .

Morcote-Ríos, Gaspar y Rodrigo Bernal

2001 "Remains of Palms (Palmae) at Archaeological Sites in the New World: A Review”, The Botanical Review, 67 (3): 309-350. New York: The New York Botanical Garden Press. 
Palma, Ricardo

1967[1877] Tradiciones Peruanas. Cuarta serie. Madrid: Espasa-Calpe.

Pearsall, Deborah, M.

2000 [1989] Paleoethnobotany: A Handbook of Procedures. San Diego: Academic Press.

Piacenza, Luigi

2001 "Los restos botánicos de la Cueva del Lazo, Ocozocoautla, Chiapas", Investigación, Revista ICACH Nueva época, 1(5): 25-38. Tuxtla Gutiérrez: Universidad de Ciencias y Artes del Estado de Chiapas.

Porras Barrenechea, Raúl

1999 Indagaciones peruanas: El legado quechua. Lima: Universidad Nacional Mayor de San Marcos, Fondo Editorial.

2000 Programa de Manejo de la Reserva de la Biosfera Selva el Ocote (PMREBISO). Comisión Nacional de Áreas Naturales Protegidas. México.

Quero, R. H.

1992 “Current status of Mexican palms”, Principes, 36 (4): 203-216. Austin: International Palm Society.

Real Academia Española

2001a Corpus diacrónico del español (CORDE), 22. edición. Madrid: Real Academia Española. En línea: <http://www.rae.es> [20 de noviembre 2012].

2001b Diccionario de la lengua española, 22. edición. Madrid: Real Academia Española. En línea: <http://www.rae.es> [20 de noviembre 2012].

Sanders, Andrew

2011 “Árboles tropicales del área maya”. Disponible en: < http://sanders5.ucr.edu/ arboles_especiesnombre.php?nombrenumero $=4304,423>$.

Varela Scherrer, Carlos Miguel

2012 "Análisis preliminar del material arqueofaunístico del sitio de Chinikihá, 1 Chiapas, temporada 2010", Informe, cuarta temporada, Proyecto Arqueológico Chinikihá, 2011. Report submitted to the Instituto Nacional de Antropología e Historia, México, Rodrigo Liendo (ed.), 1-7. Disponible en: <http://www. mesoweb.com/resources/informes/Chinikiha2011.html>. 
